\title{
DETERMINACIÓN DEL GRADO DE BIENESTAR ANIMAL EN SISTEMAS DE PRODUCCIÓN CAPRINO DEL MUNICIPIO DE OCAÑA, NORTE DE SANTANDER.
}

\author{
Determination of the degree of animal welfare in goat production \\ systems in the municipality of Ocaña, North of Santander
}

\section{Artículo de Investigación}

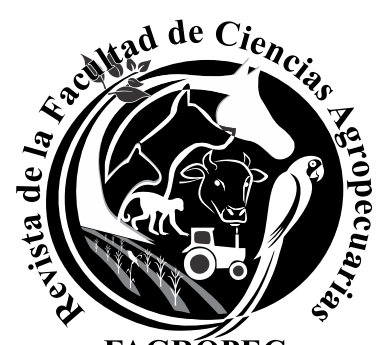

FAGROPEC

Recibido 21 de enero de 2019. Aceptado 15 de marzo de 2019.

${ }^{1}$ Universidad Francisco de Paula Santander, GI@SDy GIPAB

${ }^{2}$ Universidad Francisco de Paula Santander, GI@SDy GIPAB

${ }^{3}$ Universidad Francisco de Paula Santander Ocaña.

${ }^{4}$ Universidad Francisco de Paula Santander, GIPAB

\section{Como citar:}

HOYOS PATIÑO, Johann Fernando, et al. Determinación del grado de bienestar animal en sistemas de producción caprino del municipio de Ocaña, Norte de Santander. En: Revista Facultad Ciencias Agropecuarias FAGROPEC. Universidad de la Amazonia, Florencia - Caquetá. Volumen 11 enero-junio, 2019. Pp. 14-22. ISSN-Revista en Línea: 2539$178 \mathrm{X}$

*Autor para correspondencia: jfhoyosp@ufpso.edu.co
Johann Fernando Hoyos Patiño ${ }^{1 *}$, Daniel Antonio Hernández Villamizar ${ }^{2}$, Jhoneisson Pallares Rincón ${ }^{3}$ y Blanca Liliana Velásquez Carrascal ${ }^{4}$

\section{RESUMEN}

La presente investigación tuvo como propósito evaluar el bienestar animal en dos sistemas de producción caprinos en Ocaña Norte de Santander. El enfoque investigativo fue mixto; componente cualitativo recopilando datos por observación e interacción directa con los ejemplares, y el cuantitativo midió los cuatro principios del protocolo Welfare Quality ${ }^{\circledR}$ (buena alimentación, buen alojamiento, buena salud y comportamiento adecuado), 11 criterios y 33 indicadores para valorar el bienestar animal. El tipo de investigación aplicada fue descriptiva caracterizando el proceso de los apriscos. El análisis de resultados demostró que los dos sistemas de producción obtuveiron calificación de excelente, ya que el puntaje en todos los principios evaluados fue mayor al $55 \%$, presentado una valoración general promedio de $93.47 \%$. Discriminados de la siguiente forma $98,47 \%$ buena alimentación, $95,02 \%$ buen alojamiento, $90,20 \%$ buena sanidad y $90,18 \%$ en comportamiento apropriado, debido a los puntos a mejorar en los indicadores de los critérios evaluados.

Palabras claves:

Bienestar; Caprinos; Criterio; Indicador; Welfare Quality®.

\section{ABSTRACT}

The purpose of this research was to evaluate animal welfare in two goat production systems in Ocaña, North of Santander. The investigative approach was mixed; the aim of the qualitative component was to collect data by observation and direct interaction with the specimens. The quantitative component was to measure the four principles of the Welfare Quality ${ }^{\circledR}$ protocol (good feeding, good housing, good health and appropriate behavior), 11 criteria and 33 indicators to assess the animal welfare. The applied type of research was descriptive, characterizing the sheepfold process. The analysis of the results highlights the excellent qualification of both production systems, since the score in all the principles evaluated was greater than $55 \%$, presenting an average general assessment of $93.47 \%$. The results were distinguished in the following way: $98.47 \%$ good feeding, $95.02 \%$ good lodging, $90.20 \%$ good health and $90.18 \%$ in appropriate behavior, due to the points to be improved in the indicators of the 
evaluated criteria.

Key words:

Welfare; Goats; Criteria; Indicator; Welfare Quality®.

\section{INTRODUCCIÓN}

El concepto de bienestar animal - BA, permite una amplia discusión debido a las diversas apreciaciones y enfoques bioéticos que lo enmarcan. Sanmartín(2015), expresa que el BA es el inicio del convencimiento que los animales son seres sintientes que llegan a experimentar dolor o estrés; causar sufrimiento no es moralmente aceptable. Debido a esto, las producciones también son afectadas, tanto en calidad como en el desempeño; brindar un buen BA implica el compromiso de asegurar una buena calidad de vida, durante todo el ciclo vital del animal, desde el nacimiento hasta la muerte o el sacrificio, y que este último sea también humanitario (Sanmartín et al,2015; Bergaglio et al, 2017).

El protocolo Welfare Quality ${ }^{\circledR}$ usa las medidas o indicadores basadas en los propios animales, evaluadas en función del esfuerzo que hace este para superar las condiciones sociales y físicas presentadas por el entorno y a su vez como reflejo de su estado mental (Dalmau y Velarde, 2014).

En 2019, Welfare Quality®, detalló los 4 principios de BA para su medición: buena alimentación, buen alojamiento, buena salud y comportamiento apropiado. Dentro de estos principios, se identificaron 12 criterios diferentes (Sanmartín et al, 2015). A su vez, se seleccionó un promedio de 20 a 35 indicadores o medidas para evaluar los criterios, los cuales están basados en estudios y bibliografía científica.

La aplicación de los protocolos de evaluación de BA, fortalece los sistemas de producción pecuarios, como el caprino, facilitando la intervención temprana en los problemas que se presenten, focalizándolos y ofreciendo mejoras oportunas (Wageningen, 2012a), ante lo que surge la pregunta de: a partir de la aplicación del protocolo Welfare Quality ¿ ¿Cuál es el nivel de bienestar de los animales en los sistemas de producción caprino dedicados a la producción lechera en los apriscos de Ocaña, Norte de Santander?

\section{METODOLOGÍA}

La investigación se desarrolló en el municipio de Ocaña, ubicado en la zona Centro Occidental del departamento, y pertenece a la sub-región noroccidental, limita por el Oriente con los municipios de San Calixto, La Playa y Abrego. Por el Norte con los municipios de Teorama, Convención y El Carmen. Por el Sur con el municipio de Ábrego. Por el Occidente. Con los municipios de San Martín y Río de Oro (Alcaldía de Ocaña, 2018).

En el contexto Nacional, Ocaña hace parte del Norte de Santander, ubicado sobre la cordillera Oriental en una zona completamente montañosa, tiene una extensión aproximada de $627.72 \mathrm{Km}^{2}$ que representa el 2,76\% del área total del Departamento, la cabecera municipal se encuentra a una distancia de 203 Km. De la capital del Departamento por la vía Ocaña-Cúcuta, además se comunica 
con el Departamento del Cesar en la vía Rio de Oro-Aguachica, empalmando con la carretera que va a la Costa Atlántica y hacia el centro del país, de igual manera se comunica con la capital del Departamento por la vía Convención - Tibú - Cúcuta (Alcaldía de Ocaña, 2018).

El enfoque de la investigación fue mixto, con la integración de los métodos cualitativo y cuantitativo. Según Taylor y Bogdan (2004) el enfoque cualitativo de investigación, es el conjunto de técnicas para recopilar datos usando la observación y la interacción directa con los sujetos de estudio (caprinos), entendiendo de forma particular el proceso productivo y la interacción ambiental. Para Bernal (2016), el enfoque cuantitativo se fundamenta en la medición de las características del fenómeno. En este caso, delimitando los criterios e indicadores para valorar el (BA), utilizando el protocolo Welfare Quality® (Tabla 1) (Wageningen, 2012a).

El tipo de investigación aplicada fue descriptiva, señalando las características del fenómeno existente a través de la recolección de datos, interpretación y análisis en atención al universo real de donde proviene Arias (1999) caracterizando el proceso productivo de los apriscos.

La selección de los dos sistemas de producción (apriscos), fue amparado en el tipo de muestra no probabilística por conveniencia Balestrini (2006), el cual permite seleccionar la población objeto de estudio, dadas las características de disponibilidad de acceso, colaboración del productor y presupuesto para la ejecución de la investigación (Bernal, 2006). Y para la evaluación del BA se tuvo en cuenta los cuatro parámetros definidos por el protocolo Welfare Quality® y los 12 criterio recomendados genéricamente para evaluar el BA en sistemas de producción (Tabla 1).

Tabla 1: Parámetros utilizados en el proyecto Welfare Quality® para la evaluación del BA

\begin{tabular}{|c|c|c|}
\hline PARAMETRO & CRITERIO & INDICADORES \\
\hline Buena alimentación & $\begin{array}{l}\text { 1. Ausencia de hambre prolongada. } \\
\text { 2. Ausencia de sed prolongada }\end{array}$ & - \\
\hline \multirow[t]{2}{*}{ Buen alojamiento } & 3. Comodidad en el lugar de descanso & $\begin{array}{l}\text { Es evaluado a través de comportamientos } \\
\text { como levantarse y echarse, sin lesiones. }\end{array}$ \\
\hline & $\begin{array}{l}\text { 4. Comodidad térmica. } \\
\text { 5. Movimiento con facilidad }\end{array}$ & $\begin{array}{l}\text { La zona de descanso sin problemas de salud } \\
\text { y movimientos en su alrededor. }\end{array}$ \\
\hline \multirow[t]{3}{*}{ Buena Sanidad } & 6. Ausencia de lesiones y alopecias & $\begin{array}{l}\text { A excepción aquellos que son producidos por } \\
\text { enfermedades o intervenciones. }\end{array}$ \\
\hline & 7. Ausencia de enfermedad. & $\begin{array}{l}\text { Problemas clínicos distintos a causados por } \\
\text { lesiones. }\end{array}$ \\
\hline & $\begin{array}{l}\text { 8. Desaparición de dolor causado por } \\
\text { mal manejo. }\end{array}$ & Mutilaciones y aturdimiento. \\
\hline \multirow[t]{4}{*}{ Buen comportamiento } & 9. libre comportamiento social. & $\begin{array}{l}\text { Características positivas (lengueteo social) y } \\
\text { negativos (agresión). }\end{array}$ \\
\hline & $\begin{array}{l}\text { 10. Expresión de otros } \\
\text { comportamientos (natural). }\end{array}$ & $\begin{array}{l}\text { Aspectos positivos (exploración) y negativos } \\
\text { (conductas estereotipadas). }\end{array}$ \\
\hline & 11. Relación adecuada humano-animal. & Sin miedo a los humanos. \\
\hline & 12. Ausencia de miedo & A excepción de miedo hacia personas. \\
\hline
\end{tabular}

Definición de criterios para la evaluación global del BA. Botreau, et al., 2007, adaptado por Pallares, 2019; Hoyos et al., 2019 
Para la investigación fueron seleccionados dos sistemas, el primero, SP1, ubicado en la Granja Experimental de la Universidad Francisco de Paula Santander sede Ocaña vía Acolsure sobre el Algodonal. La altura del aprisco es de 1150 m.s.n.m., esta zona cuenta con un área de 3500 metros cuadrados, con una temperatura promedio de $23^{\circ} \mathrm{C}$, la humedad relativa es del $70 \%$, con una precipitación de $1000 \mathrm{~mm}$ anuales. Cuenta con un total de 96 animales divididos de la siguiente forma: 33 cabras adultas, 45 cabretonas, 3 levante, 5 machos reproductores y 10 crías, todos estos de las razas, Canaria, Toggenburg, Anglonubiana, Alpina, Criolla Santandereana, Saanen y sus cruces. Sistema de producción semiestabulado y utilizan ordeño mecánico.

El segundo, SP2, ubicado en la vereda Pueblo Nuevo del municipio de Ocaña, al occidente de este municipio. La altura del aprisco es de 1671 m.s.n.m., con una temperatura promedio de $19,4^{\circ} \mathrm{C}$ y una precipitación de $1241 \mathrm{~mm}$ anuales. Al momento de realizar el estudio, se cuenta con un inventario animal de 86 caprinos divididos de la siguiente manera: 34 cabras, 20 cabretonas, 2 machos reproductores y 10 crías, todos estos de las razas, Sannen, Alpina francesa, Toggenburg y algunos cruces de los mismos. Sistema de producción semiestabulado con ordeño manual.

La alimentación en los dos sistemas se basa en praderas con una combinación entre Pará (Brachiaria mutica) y Guinea (Panicum máximum), pasto de corte Cuba 22 (Ct-115), Taiwán (Pennisetum purpureum), King grass (Pennisetum sp), Maíz (Zea mays) y bancos forrajeros como botón de oro (Tithonia diversifolia). El promedio de productividad de leche en estos SP, es de 8 a 15 litros/ día. La reproducción del SP1, se realiza por medio de inseminación artificial a tiempo fijo (IATF) y transferencia de embriones (TE), y uso de macho para las hembras que repiten celo, en el SP2 se usa monta natural.

Para la medición en campo del BA, se aplicaron indicadores que evaluaron el bienestar basados en el animal y otros indicadores basados en el ambiente, los cuales evalúan los criterios y principios del protocolo Welfare Quality ${ }^{\circledR}$ (Wageningen, 2012b). Para determinar la calificación de cada principio se promedian las calificaciones obtenidas en cada criterio, las cuales son resultado del promedio de cumplimento de cada indicador (Pallares, 2019; Hoyos et al, 2019).

$\% \mathrm{CC}$ : Porcentaje cumplimento del criterio de bienestar.

$\% \mathrm{CC}=\left[\sum \%\right.$ Cumplimento Indicador de bienestar $] /$ (número de indicadores evaluados)

\%CP: Porcentaje cumplimento del principio

$\% \mathrm{CP}=\left[\sum \%\right.$ Cumplimiento criterio de bienestar $] /$ (número de criterios evaluados)

Los datos se promediaron para calcular los puntajes de los criterios y los principios. Seguidamente, se asignó la categoría de bienestar, de acuerdo a la siguiente escala (Wageningen, 2012b, adaptada por Pallares, 2019; Hoyos et al, 2019):

- Excelente (80 puntos): el bienestar de los animales está en el nivel más alto.

- Elevado (55 puntos): el bienestar de los animales es bueno.

- Aceptable (20 puntos): el bienestar está sobre o cumple con los requerimientos mínimos.

- No clasificado: el bienestar es bajo y considerado inaceptable. 
La evaluación del BA en aprisco se considerada excelente si los puntajes en todos los principios es mayor a 55\% y tiene valores mayores a $80 \%$ en dos de ellos. Elevada, si los puntajes son mayores de $20 \%$ en todos los principios y obtiene valores mayores a $55 \%$ en dos de ellos. Y Aceptable cuando cuenta con puntajes mayores que $10 \%$ en todos los principios y más de $20 \%$ en tres de ellos (Wageningen, 2012a; Pallares, 2019; Hoyos et al, 2019)

Para la investigación no fueron manipulados de manera directa los animales, sino los registros de los sistemas productivos y mediciones de componentes ambiéntelas, razón por la cual no se hizo necesario el requerimiento de aval de un comité de ética.

\section{RESULTADOS Y DISCUSIÓN}

Para cada uno de los sistemas estudiados, fueron evaluados 11 criterios, con 19 indicadores medidos en los animales y 9 de tipo medio ambiental (Tabla 2)

El parámetro de buena alimentación se evalúo con 2 criterios: ausencia de hambre se midió con 2 criterios condición corporal y condición de pelaje; para el criterio ausencia de sed se evalúo con 2 criterios; cantidad suficiente de beberos y condición de bebederos. La diferencia en puntuación para los dos sistemas no fue relevante, presentando promedios de puntuación mayores a $98 \%$, con puntos a mejorar para el SP2 en relación al criterio de condición de bebederos con una evaluación de 91\%.

En el parámetro de buen alojamiento se evaluaron 3 criterios: libres de incomodidad en el descanso, con 5 criterios; condición térmica con 1 criterio y facilidad de movimiento con 3 criterios. Se presentaron diferencias para el primer criterio con puntos a mejorar en el indicador de condición de pisos y limpieza de pisos para el SP2 con calificación de 50\%, y para el SP1 con ponderación de 80\%, reflejando deficiencias en el criterio de libres de incomodidad en el descanso para los dos SP. Los 2 criterios restantes presentaron similitud en el rendimiento de calificación, mostrando rendimientos por encima de $99 \%$.

Como punto adicional para la medición de este parámetro, en el criterio de condición térmica, se registró la temperatura máxima y mínima en los dos sistemas de producción durante 16 días, dando como resultado un promedio de temperatura para el SP1 de $21,9^{\circ} \mathrm{C}$ con un variación durante el día de $6,23^{\circ} \mathrm{C}$, y para el SP2 de $20,1^{\circ} \mathrm{C}$ con un variación durante el día de $3,42^{\circ} \mathrm{C}$, que se ubican dentro del rango de 13 a $32^{\circ} \mathrm{C}$ reportado con Arauz (2009), citado por Pallares (2019), para cumplir con el BA.

Para el parámetro buena sanidad se calificaron 3 criterios: ausencia de lesiones con 3 indicadores; ausencia de enfermedades con 6 indicadores y ausencia de dolor inducido por manejo con 3 indicadores. Durante el estudio de identificaron puntos a mejorar para los indicadores de presencia de ubres simétricas para el SP1 y para el indicador de malas instalaciones 50\% y descorne adecuado 20\% para el SP2. Los criterios restantes para este parámetro presentaron rangos superiores a 94\% de cumplimento para ofrecer BA en los apriscos.

Finalmente, para el parámetro de buen comportamiento se evaluaron 3 criterios: libertad de compartimento social, evaluado con el criterio presencia de conductas agonísticas (evaluado por observación de comportamientos al momento de alimentarse y en el proceso de ordeño). Para el 
Tabla 2. Tabla de parámetros, criterios e indicadores con su respectiva calificación de los dos sistemas de producción caprinos evaluados.

Sistema de producción 1 (SP1)

Sistema de producción 2 (SP2)

(SP1) (SP 2) $\quad$ (SP1) $\quad$ (SP 2) $\quad$ (SP1)

(SP 2)

\begin{tabular}{|c|c|c|c|c|c|c|c|}
\hline \multicolumn{8}{|c|}{ Buena alimentación } \\
\hline Indicador & Criterio & $\begin{array}{l}\text { Calif. } \\
\text { Indiv }\end{array}$ & $\begin{array}{l}\text { Calif. } \\
\text { Indiv }\end{array}$ & $\begin{array}{l}\text { Calif. } \\
\text { General }\end{array}$ & $\begin{array}{l}\text { Calif. } \\
\text { General }\end{array}$ & $\begin{array}{l}\text { Calif. } \\
\text { Global } \\
\end{array}$ & $\begin{array}{l}\text { Calif. } \\
\text { Global } \\
\end{array}$ \\
\hline $\begin{array}{l}\text { Condición corporal } \\
\text { Condición de pelaje }\end{array}$ & Ausencia de hambre & $\begin{array}{l}100 \% \\
98 \%\end{array}$ & $\begin{array}{l}100 \% \\
98 \%\end{array}$ & $99 \%$ & $99 \%$ & $99,58 \%$ & $97,35 \%$ \\
\hline $\begin{array}{l}\text { Cantidad suficiente de bebederos } \\
\text { Condición de bebederos }\end{array}$ & Ausencia de sed & $\begin{array}{l}100 \% \\
100 \%\end{array}$ & $\begin{array}{l}100 \% \\
91 \%\end{array}$ & $100 \%$ & $96 \%$ & & \\
\hline
\end{tabular}

Condición de bebederos

\begin{tabular}{|c|c|c|c|c|c|c|c|}
\hline \multicolumn{8}{|c|}{ Buen alojamiento } \\
\hline Indicador & Criterio & $\begin{array}{l}\text { Calif. } \\
\text { Indiv }\end{array}$ & $\begin{array}{l}\text { Calif. } \\
\text { Indiv }\end{array}$ & $\begin{array}{l}\text { Calif. } \\
\text { General }\end{array}$ & $\begin{array}{l}\text { Calif. } \\
\text { General }\end{array}$ & $\begin{array}{l}\text { Calif. } \\
\text { Global }\end{array}$ & $\begin{array}{l}\text { Calif. } \\
\text { Global } \\
\end{array}$ \\
\hline Condición pisos & $\begin{array}{l}\text { Libres de incomodidad } \\
\text { en el descanso }\end{array}$ & $80 \%$ & $50 \%$ & $91 \%$ & $80 \%$ & $96,82 \%$ & $93,21 \%$ \\
\hline Limpieza de pisos & & $80 \%$ & $50 \%$ & & & & \\
\hline Limpieza de animales & & $96 \%$ & $98 \%$ & & & & \\
\hline Ventilación del aprisco & & $100 \%$ & $100 \%$ & & & & \\
\hline Presencia de zona de aislamiento & & $100 \%$ & $100 \%$ & & & & \\
\hline Rango de temperatura óptimo & Condición térmica & $100 \%$ & $100 \%$ & $100 \%$ & $100 \%$ & & \\
\hline Carga animal en el aprisco & $\begin{array}{l}\text { Facilidad de } \\
\text { movimiento }\end{array}$ & $100 \%$ & $100 \%$ & $99 \%$ & $100 \%$ & & \\
\hline Acceso a pasturas & & $100 \%$ & $100 \%$ & & & & \\
\hline Sin arrodillamiento al comer & & $98 \%$ & $100 \%$ & & & & \\
\hline \multicolumn{8}{|c|}{ Buena sanidad } \\
\hline Indicador & Criterio & $\begin{array}{l}\text { Calif. } \\
\text { Indiv }\end{array}$ & $\begin{array}{l}\text { Calif. } \\
\text { Indiv }\end{array}$ & $\begin{array}{l}\text { Calif. } \\
\text { General }\end{array}$ & $\begin{array}{l}\text { Calif. } \\
\text { General }\end{array}$ & $\begin{array}{l}\text { Calif. } \\
\text { Global }\end{array}$ & $\begin{array}{l}\text { Calif. } \\
\text { Global }\end{array}$ \\
\hline Sin presencia de cojera severa & Ausencia de lesiones & $100 \%$ & $100 \%$ & $95 \%$ & $98 \%$ & $95,68 \%$ & $84,71 \%$ \\
\hline Sin abscesos en el cuerpo & & $100 \%$ & $100 \%$ & & & & \\
\hline Presencia de ubre simétrica & & $86 \%$ & $94 \%$ & & & & \\
\hline Sin secreción nasal & $\begin{array}{l}\text { Ausencia de } \\
\text { enfermedades }\end{array}$ & $100 \%$ & $100 \%$ & $98 \%$ & $99 \%$ & & \\
\hline Sin secreción ocular & & $100 \%$ & $100 \%$ & & & & \\
\hline Sin suciedad fecal & & $96 \%$ & $97 \%$ & & & & \\
\hline Sin dificultad al respirar & & $100 \%$ & $100 \%$ & & & & \\
\hline Sin presencia de tos & & $100 \%$ & $100 \%$ & & & & \\
\hline Sin presencia de mastitis & & $95 \%$ & $100 \%$ & & & & \\
\hline Descorné adecuado & $\begin{array}{l}\text { Ausencia de dolor } \\
\text { inducido por manejo }\end{array}$ & $100 \%$ & $20 \%$ & $93 \%$ & $57 \%$ & & \\
\hline Recorte de pezuñas & & $100 \%$ & $100 \%$ & & & & \\
\hline Estado de Instalaciones & & $80 \%$ & $50 \%$ & & & & \\
\hline \multicolumn{8}{|c|}{ Buen comportamiento } \\
\hline Indicador & Criterio & $\begin{array}{l}\text { Calif. } \\
\text { Indiv }\end{array}$ & $\begin{array}{l}\text { Calif. } \\
\text { Indiv } \\
\end{array}$ & $\begin{array}{l}\text { Calif. } \\
\text { General } \\
\end{array}$ & $\begin{array}{l}\text { Calif. } \\
\text { General } \\
\end{array}$ & $\begin{array}{l}\text { Calif. } \\
\text { Global } \\
\end{array}$ & $\begin{array}{l}\text { Calif. } \\
\text { Global } \\
\end{array}$ \\
\hline $\begin{array}{l}\text { Conductas agonísticas } \\
\text { (golpes, etc) }\end{array}$ & $\begin{array}{l}\text { Libertad de } \\
\text { comportamiento social }\end{array}$ & $86 \%$ & $95 \%$ & $86 \%$ & $95 \%$ & $88,81 \%$ & $91,55 \%$ \\
\hline Sin presencia de aislamiento & $\begin{array}{l}\text { Expresión de otros } \\
\text { comportamientos }\end{array}$ & $100 \%$ & $100 \%$ & $100 \%$ & $100 \%$ & & \\
\hline Latencia a prueba de contacto & $\begin{array}{l}\text { Relación adecuada } \\
\text { humano-animal }\end{array}$ & $80 \%$ & $80 \%$ & $80 \%$ & $80 \%$ & & \\
\hline
\end{tabular}


criterio expresión de otros comportamientos, calificado con el indicador presencia de aislamiento (revisión de animales aislados de lote por motivos marginamiento social) y para el criterio relación adecuada humano-animal, con el criterio latencia a prueba de contacto (realizando la prueba de los $30 \mathrm{seg}$ antes de entrar al corral con el evaluador inmóvil y 3 minutos dentro del corral) midiendo la cantidad de animales del lote que se acercan y el tiempo de respuesta.

Estas pruebas de comportamiento dieron como resultado puntos a mejorar en el criterio de conductas agonista (presencia de golpes entre los animales y jerarquización) para el SP1, que de debió a que muchos de sus ejemplares cuentan con cuernos prominentes. Para los demás criterios de comportamiento los dos SP cumplen con parámetros superiores a $80 \%$ para brindar BA en los SP.

La calificación global para determinar el nivel de BA en los SP fue de 95,22\% para el SP1 y 91,71\% para el SP2 registrando una categoría de excelente para los dos aprisco. Es de anotar la diferencia marcada entre los dos SP en el parámetro de buena sanidad, donde se presento una diferencia de 10,97 puntos porcentuales, debido a la calificación de 57\% para el criterio de ausencia de dolor inducido por manejo donde se evaluaron indicadores deficientes en descorne adecuado y malas instalaciones. Para fortalecer estos indicadores se diseño un plan de mejorar con el productor para el proceso de descorne y mejoramiento de pisos e instalaciones en general.

Para los parámetros de buena alimentación la diferencia porcentual entre los dos SP fue de 2,23 puntos y de 3,61 para el de buen alojamiento (Figura 1). En el único criterio donde el SP2 presento mayores indices de cumplimiento que el SP1 fue en el criterio de buen comportamiento, con una diferencia porcentual de 2,74. Cabe resaltar, que este parámetro en el promedio general presento el nivel de calificación menor con 90,18\%.

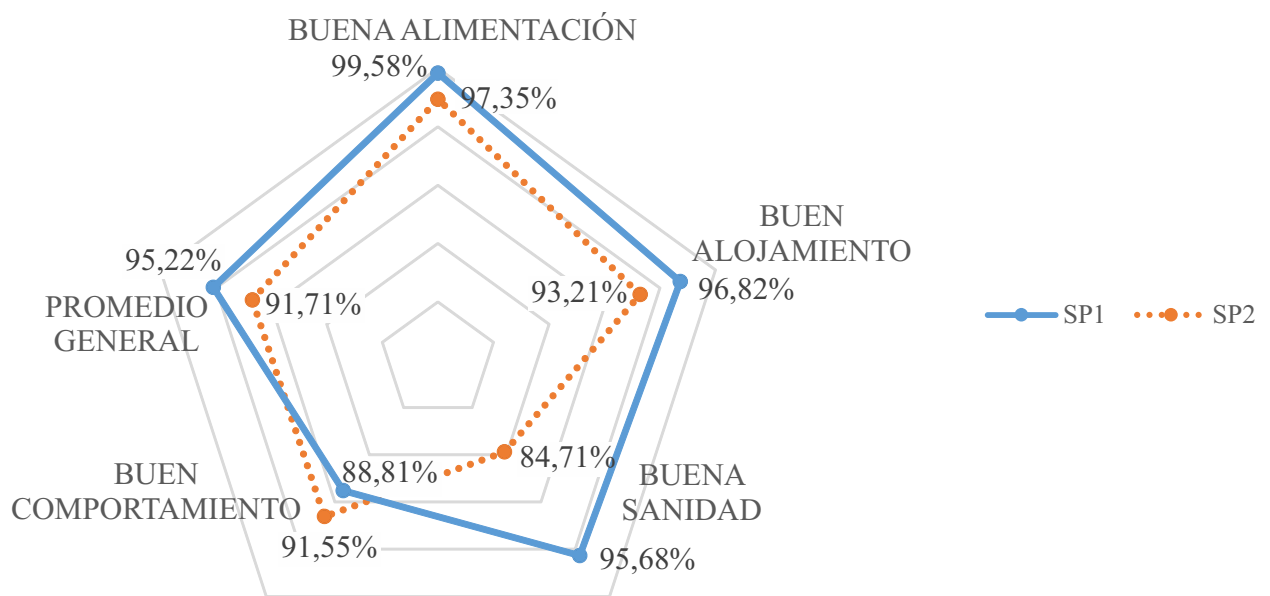

Figura 1. Resultados ponderados para los cuatro parámetros evaluados y promedio general obtenido en la evaluación.

\section{CONCLUSIONES}

La evaluación del BA del SP1, a partir de la instauración del protocolo Welfare Quality ${ }^{\circledR}$, presentó un porcentaje final por encima de 95\%, ubicándose en categoría de excelente, con puntos a mejorar en 
indicadores como: condición y limpieza de pisos; incidencia de mastitis; presencia de conductas agonísticas de algunos animales, debido a la presencia de cuernos largos y latencia a la prueba de contacto con humanos.

EL SP2 obtuvo evaluación por encima de 90\%, encontrándose en la categoría de excelente. De la misma manera, con puntos a mejorar en indicadores como condición de bebederos, condición y limpieza de pisos, deficiencias en el descorné, estado de instalaciones, presencia conductas agonísticas y latencia a la prueba de contacto con el humano.

El proceso productivo de los apriscos, presenta diferencias que no incidente en la medición de los parámetros de BA. Estos protocolos de evaluación, deben ser apropiados por los operarios, para que el ciclo de evolución y ajuste sea constante.

La instauración del protocolo Welfare Quality ${ }^{\circledR}$, para la medición del nivel de bienestar de los animales en los sistemas de producción caprino dedicados a la producción lechera en los apriscos de Ocaña, Norte de Santander, ofreció indicaciones precisas para la mejora del sistema, constituyéndose en una herramienta efectiva y de fácil uso.

\section{LITERATURA CITADA}

ALCALDÍA DE OCAÑA. Sitio oficial Alcaldía de Ocaña - Norte de Santander. 2018. \{En línea . Disponible en: https://cutt.ly/Fyn3eK3

ARAÚZ, Edil Enrique. Importancia del microambiente para el desempeño fisiológico y efectos negativos del estrés calórico sobre la capacidad fisiológica y de producción en los caprinos y ovinos. Dpto. de Zootecnia, Ftad de Ciencias Agropecuarias, Universidad de Panamá. 2009. \{En línea\}. Disponible en: https://cutt.ly/dyn304e

ARIAS, F. El proyecto de investigación. Guía para su elaboración. Caracas: Episteme, $3^{\circ}$ Ed.1999

BALESTRINI, M. Cómo se elabora el Proyecto de Investigación. Caracas, Venezuela: BL Consultores Asociados.2006.Pp. 62-123

BERGAGLIO, J.P., PALAU, H., \& SENESI S.I. Instrumentación de un protocolo de BA y su impacto sobre una explotación ganadera en la provincia de buenos aires. Agronomía \& Ambiente. Revista de la Facultad de Agronomía ISSN 2344-9039 (en línea) - ISSN 2314-2243 (impreso). 2017. \{En línea\}. Disponible en: https://cutt.ly/qyn91or

BERNAL, C. Metodología de la investigación. Bogotá: Pearson, $4^{\circ}$ Ed.2016

DALMAU, Antoni., \& VELARDE, Antonio.Protocolo Welfare Quality®. Evaluación del (BA).2014. \{En línea\}. Disponible en: https://bit.ly/2Lvvzll

HOYOS PATIÑO, Johann., BERMÚDEZ GUTIÉRREZ, Edinson., HERNÁNDEZ VILLAMIZAR, Daniel., \& VELÁSQUEZ CARRASCAL, Blanca. Aplicación del protocolo Welfare Quality ${ }^{\circledR}$ en criaderos equinos para determinar el grado de BA. Mundo FESC, 9(18). Pp. 24-30. 2019. \{En línea\}. Disponible en: https://cutt.ly/iyn3VlB 
PALLARES RINCON, Jhoneisson. Evaluación del BA en el sistema de producción caprino de la granja experimental de la Universidad Francisco de Paula Santander Ocaña y la finca la Variante ubicada en la vereda pueblo nuevo del municipio de Ocaña, mediante el protocolo Welfare Quality ${ }^{\circledR}$. Trabajo de grado. Director: Johann Fernando Hoyos Patiño. Facultad de ciencias agaririas y del ambiente. Universidad Francisco de Paula Santander Ocaña. 2019. \{En línea\}.Disponible en: https://cutt.ly/ayn3Pce

SANMARTÍN SANCHEZ, Lourdes., PEREA, Jose., BLANCO PENEDO, Isabel., PÉREZ RICO, Almudena., \& VEGA PLA, Jose Luis. (BA) en equinos (Equus caballus): una evaluación comparativa en reproductores del sur de España. Revista Científica, XXV (6). 2015. Pp. 471-480. \{En línea\}. Disponible en: https://bit.ly/2kpC79t

TAYLOR, S., \& BOGDAN, R. El proceso de la investigación científica. Mexico: Limusa. 2004

WAGENINGEN, U. R. Welfare Monitoring System Horses - Calculation of scores-version 2.0 Wageningen UR Livestock Research, 44. 2012b. \{En línea\}. Disponible en: https://edepot.wur.n1/238620

WAGENINGEN, U. R.Welfare Monitoring System-Assessment protocol for horses, version 2.0. Wageningen UR Livestock Research, 44. 2012a. \{En línea\}. Disponible en: https://edepot.wur.nl/238619

WELFARE QUALITY CONSORTIUM. Certificación IRTA en (BA) "basada en Welfare Quality”. Lelystad, The Netherlands. 2019. \{En línea\}. Disponible en: https://bit.ly/2O3871f 